\title{
C-complete sets for compromise stable games
}

\author{
by \\ Trine Tornøe Platz, \\ Herbert Hamers \\ and \\ Marieke Quant
}

Discussion Papers on Business and Economics

No. 25/2012

\author{
FURTHER INFORMATION \\ Department of Business and Economics \\ Faculty of Social Sciences \\ University of Southern Denmark \\ Campusvej 55 \\ DK-5230 Odense M \\ Denmark \\ Tel.: +4565503271 \\ Fax: +4565503237 \\ E-mail:1ho@sam.sdu.dk \\ http://www.sdu.dk/ivoe
}

ISBN 978-87-91657-78-8 


\title{
C-complete sets for compromise stable games
}

\author{
Trine Tornøe Platz ${ }^{1} \quad$ Herbert Hamers ${ }^{2} \quad$ Marieke Quant ${ }^{2}$
}

\begin{abstract}
The core cover of a TU-game is a superset of the core and equals the convex hull of its larginal vectors. A larginal vector corresponds to an ordering of the players and describes the efficient payoff vector giving the first players in the ordering their utopia demand as long as it is still possible to assign the remaining players at least their minimum right. A game is called compromise stable if the core is equal to the core cover, i.e. the core is the convex hull of the larginal vectors. This paper analyzes the structure of orderings corresponding to larginal vectors of the core cover and conditions ensuring equality between core cover and core. We introduce compromise complete (or c-complete) sets that satisfy the condition that if every larginal vector corresponding to an ordering of the set is a core element, then the game is compromise stable. We use combinatorial arguments to give a complete characterization of these sets. More specifically, we find c-complete sets of minimum cardinality and a closed formula for the minimum number of orderings in c-complete sets.
\end{abstract}

Keywords: Core, core cover, larginal vectors.

\section{JEL Classification Number: C71.}

\footnotetext{
${ }^{1}$ Department of Business and Economics, University of Southern Denmark, Campusvej 55, DK5230 Odense M, Denmark

${ }^{2}$ Department of Econometrics \& OR and CentER, Tilburg University, P.O. Box 90153, 5000 LE, Tilburg, The Netherlands.Corrresponding author: H.J.M.Hamers@tilburguniversity.edu
} 


\section{Introduction}

The core, introduced by Gillies (1953), is a well-established solution concept for TU-games and equals the set of efficient allocations that satisfy the property that no subcoalition has an incentive to leave the grand coalition and act on their own. The Weber set (Weber (1988)) and the core cover (Tijs and Lipperts (1982)) are well-known supersets of the core.

The Weber set is the convex hull of the marginal vectors. A marginal vector corresponds to an ordering of the players and is the efficient allocation vector that assigns to every player his marginal contribution to the coalition consisting of players preceding him in the ordering. Shapley (1971) and Ichiishi (1981) showed that a TU-game is convex if and only if the core is equal to the Weber set, i.e., if the core is the convex hull of the marginal vectors.

A set of marginal vectors characterizes convexity if it satisfies the condition that a game is convex whenever all marginal vectors of this set are core elements. Rafels and Ybern (1995) showed that the set consisting of either all even or all odd marginal vectors are sets that characterize convexity. Van Velzen et al. (2002) improved this result by finding such characterizing sets with a smaller cardinality by using a neighbor argument showing that if two consecutive neighbors of a marginal vector are in the core, so is the marginal vector itself. Using combinatorial arguments Van Velzen et al. (2004) derive the minimum cardinality of sets that characterize convexity.

Quant et al. (2005) showed that the core cover equals the convex hull of the larginal vectors. A larginal vector corresponds to an ordering of the players and equals the efficient payoff vector giving the first players in the ordering their utopia demand as long as it is still possible to assign the remaining players at least their minimum right. A TU-game is compromise stable if and only if the core cover equals the core, i.e., if the core is the convex hull of the larginal vectors. The interest in compromise stable games is two-fold. In many TU-games the nucleolus (Schmeidler (1969)) is hard or even impossible to compute, but for the class of compromise stable games, Quant et al. (2005) provide a closed formula for the nucleolus. Moreover, 
the class of compromise stable games contains several interesting classes of games such as clan games (Potters et al. (1989)), big boss games (Muto et al. (1989)), 1-convex games (Driessen(1988)) and bankruptcy games (Curiel et al. (1988)). In fact, the class of bankruptcy games is the intersection between the classes of convex and compromise stable games. This means that any game that is both convex and compromise stable is strategically equivalent to a bankruptcy game.

In this paper we investigate the relation between compromise stability of TU games and larginal vectors. More precisely, we study the structure of the generators, i.e. the orderings corresponding to larginal vectors, of the core cover and conditions ensuring that the game is compromise stable.

A set of orderings is called c-complete if each game for which the larginal vectors corresponding to this set are core elements is compromise stable. First, we show that the sets of all even and odd orderings are c-complete sets. Second, by using the specific nature of larginal vectors along with combinatorial arguments, we are able to exactly identify the minimum cardinality of c-complete sets.

The paper is organized as follows: Section 2 presents some notation. In section 3, we start by showing that the sets of all even and all odd orderings are c-complete sets. Subsequently, the main results are presented: we present a necessary and sufficient condition for a set of orderings to be c-complete and determine the minimum cardinality of c-complete sets.

\section{Preliminaries}

A transferable utility game (TU-game) is a pair $(N, v)$, where $N=\{1, \ldots, n\}$, the grand coalition, is a finite set of players and $v: 2^{N} \rightarrow \mathbb{R}$ is a function that assigns to every coalition $S \subseteq N$ a worth $v(S)$, with $v(\emptyset)=0$. We often refer to a game as $v$ rather than $(N, v)$ when no confusion can arise. The set of transferable utility games with player set $N$ is denoted by $T U^{N}$.

For $k \in\{1, \ldots, n\}, \mathcal{S}_{k}$ denotes the set of all coalitions with cardinality $k$, i.e., $S_{k}=\left\{S \in 2^{N}|| S \mid=k\right\}$.

Let $N$ be a finite set of players. An ordering is a bijective function 
$\sigma:\{1, \ldots,|N|\} \rightarrow N$. The set of all orderings is denoted $\Pi(N)$, and $\sigma(h)$ denotes the player at position $h$ in the ordering $\sigma$. An ordering $\sigma_{h}, h \in$ $\{1, \ldots, n-1\}$, denotes the $h$ 'th neighbor of $\sigma$ which is obtained by switching players at positions $h$ and $(h+1)$ in $\sigma$. Thus,

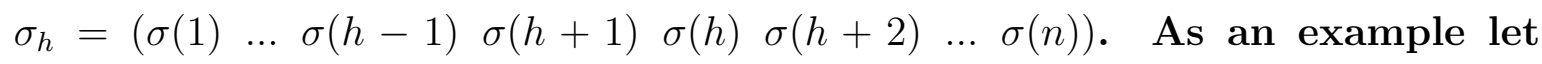
$N=\{1,2,3,4\}$. If $\sigma=(1234)$ we get $\sigma_{1}=(2134), \sigma_{2}=(1324)$, and $\sigma_{3}=(1243)$.

Let the identity ordering $e$ be the ordering such that $e(i)=i$ for all $i \in N$. Then an even ordering is an ordering that can be obtained from $e$ by switching positions of neighboring players an even number of times. An ordering that is not even is called odd. The neighbor of an odd ordering is even and vice versa.

Let $\sigma \in \Pi(N)$ be an ordering and let $k \in\{1, \ldots, n\}$. Then the $k$-head of $\sigma$ refers to the first $k$ positions of $\sigma$ and the $k$-tail of $\sigma$ refer to the last $k$ positions of $\sigma$. Further, denote the set of players belonging to the $k$-head of $\sigma$ by $H_{k}^{\sigma}=\{\sigma(1), \ldots, \sigma(k)\}$, hence $H_{l}^{\sigma} \subseteq H_{k}^{\sigma}$ if $l \leq k$. Likewise, $T_{k}^{\sigma}=\{\sigma(n-k+1), \ldots, \sigma(n)\}$ denotes the set of players belonging to the $k$-tail of $\sigma$, and $T_{l}^{\sigma} \subseteq T_{k}^{\sigma}$ if $l \leq k$.

The core of a game $v$ is defined by

$$
C(v)=\left\{x \in \mathbb{R}^{N} \mid \sum_{i=1}^{n} x_{i}=v(N), \sum_{i \in S} x_{i} \geq v(S) \text { for all } S \subseteq N\right\}
$$

and describes the set of efficient allocation vectors such that no coalition has an incentive to split off from the grand coalition. The core of a game may be empty.

The utopia demand of player $i \in N$ is given by

$$
M_{i}(v)=v(N)-v(N \backslash\{i\})
$$

and describes the maximum amount player $i$ can achieve from cooperation, since the coalition consisting of the rest of the players will never settle for less than $v(N \backslash\{i\})$.

Player $i$ can gather a coalition by promising the rest of the players in the coalition their utopia demand. The maximum amount $i$ can obtain in this 
way from some coalition is denoted the minimum right of player $i$ :

$$
m_{i}(v)=\max _{S \subseteq N: i \in S}\left\{v(S)-\sum_{j \in S \backslash\{i\}} M_{j}(v)\right\} .
$$

The core cover of a game $v$ equals

$$
C C(v)=\left\{x \in \mathbb{R}^{N} \mid \sum_{i=1}^{n} x_{i}=v(N), m(v) \leq x \leq M(v)\right\}
$$

and thus gives the set of all efficient allocation vectors such that players receive at least their minimum right but no more than their utopia demand. Observe that the core is always a subset of the core cover (cf. Tijs and Lipperts (1982)). A game $v \in T U^{N}$ is said to be compromise admissible if $m(v) \leq M(v)$ and $\sum_{i \in N} m_{i}(v) \leq v(N) \leq \sum_{i \in N} M_{i}(v)$, that is, if the core cover is non-empty. The class of compromise admissible games with player set $N$ is denoted $C A^{N}$.

The core cover equals the convex hull of the larginal vectors of a game $v \in C A^{N}$. Let $v \in C A^{N}$ and $\sigma \in \Pi(N)$. The larginal vector $l^{\sigma}(v)$ is defined by

$$
l_{\sigma(k)}^{\sigma}(v)=\left\{\begin{array}{c}
M_{\sigma(k)}(v) \text { if } \sum_{j=1}^{k} M_{\sigma(j)}(v)+\sum_{j=k+1}^{n} m_{\sigma(j)}(v) \leq v(N) \\
m_{\sigma(k)}(v) \text { if } \sum_{j=1}^{k-1} M_{\sigma(j)}(v)+\sum_{j=k}^{n} m_{\sigma(j)}(v) \geq v(N) \\
v(N)-\sum_{j=1}^{k-1} M_{\sigma(j)}(v)-\sum_{j=k+1}^{n} m_{\sigma(j)}(v) \text { otherwise }
\end{array}\right.
$$

for each $k \in\{1, \ldots, n\}$. For each ordering $\sigma \in \Pi(N)$ the larginal vector $l^{\sigma}(v)$ is the efficient payoff vector that assigns the utopia demand to the first players in $\sigma$ as long as it is still possible to give the remaining players their minimum rights. The first player that does not receive his utopia demand is called the pivot player. A larginal $l^{\sigma}(v)$ is called even (odd) if the corresponding ordering $\sigma$ is even (odd). Furthermore, $l^{\sigma_{h}}(v)$ is said to be a neighbor of $l^{\sigma}(v)$, whenever $\sigma_{h}$ is a neighbor of $\sigma$.

The following theorem is a straightforward consequence of the results of Quant et al. (2005).

Theorem 2.1. Let $v \in C A^{N}$. Then the following statements are equivalent.

(i) $v$ is compromise stable, 
(ii) $C(v)=C C(v)$,

(iii) $C(v)=\operatorname{conv}\left\{l^{\sigma}(v) \mid \sigma \in \Pi(N)\right\}$,

(iv) $v(S) \leq \max \left\{\sum_{i \in S} m_{i}(v), v(N)-\sum_{i \in N \backslash S} M_{i}(v)\right\}$ for all $S \subseteq N$.

\section{Characterizing compromise stable games using larginal vectors}

In this section, we describe c-complete sets. First, we use a neighbor argument to show that the set of even and odd orderings are c-complete sets. Second, we combine combinatorial arguments to generate c-complete sets of minimum cardinality.

We start by introducing formally the notion of compromise-complete (or c-complete) sets. A set $A \subseteq \Pi(N)$ is called c-complete if every game $(N, v) \in C A^{N}$ for which $l^{\sigma}(v) \in C(v)$ for every $\sigma \in A$ is compromise stable.

Obviously, the full set of orderings $\Pi(N)$ is a c-complete set. We will show, using a neighbor argument on orderings, that also the odd and even orderings are c-complete sets. We will show that in case all even (odd) larginals are in the core, than also all odd (even) larginals are in the core. In fact, we show that if two consecutive neighbors of an ordering correspond to larginal vectors that are in the core, then also the larginal vector corresponding to this ordering is in the core.

Lemma 3.1. Let $v \in C A^{N}, n \geq 3$, and $\sigma \in \Pi(N)$. Suppose there is an $h \in\{1, \ldots, n-2\}$ such that $l^{\sigma_{h}}(v), l^{\sigma_{h+1}}(v) \in C(v)$. Then $l^{\sigma}(v) \in C(v)$.

Proof. Since $l^{\sigma}(v)$ is by definition efficient, we only have to show that $\sum_{i \in S} l_{i}^{\sigma}(v) \geq v(S)$ for all $S \subset N$. We distinguish between three cases depending on the position of the pivot player.

Case 1. The pivot player in $l^{\sigma}(v)$ is at position $j \in\{1, \ldots, h\}$. Therefore, $l_{i}^{\sigma}(v)=$ $l_{i}^{\sigma_{h+1}}(v)$ for all $i \in N$, and

$$
\sum_{i \in S} l_{i}^{\sigma}(v)=\sum_{i \in S} l_{i}^{\sigma_{h+1}}(v) \geq v(S)
$$


for all $S \subset N$, where the inequality holds since $l^{\sigma_{h+1}}(v) \in C(v)$.

Case 2. The pivot player in $l^{\sigma}(v)$ is at position $j \in\{h+2, \ldots, n\}$. It follows that $l_{i}^{\sigma}(v)=l_{i}^{\sigma_{h}}(v)$ for all $i \in N$, and therefore

$$
\sum_{i \in S} l_{i}^{\sigma}(v)=\sum_{i \in S} l_{i}^{\sigma_{h}}(v) \geq v(S)
$$

for all $S \subset N$, where the inequality holds since $l^{\sigma_{h}}(v) \in C(v)$.

Case 3. The pivot player in $l^{\sigma}(v)$ is at position $h+1$. Here, we distinguish between two cases depending on whether the pivot player $\sigma(h+1)$ belongs to $S$ or not.

Case 3a. $\sigma(h+1) \in S$. Since $\sigma(h+1)$ is the pivot player it follows from the definition of the larginal vector that

$$
l_{\sigma(h+1)}^{\sigma}(v)+l_{\sigma(h+2)}^{\sigma}(v)=l_{\sigma(h+1)}^{\sigma_{h+1}}(v)+l_{\sigma(h+2)}^{\sigma_{h+1}}(v)
$$

and that $l_{i}^{\sigma}(v)=l_{i}^{\sigma h+1}(v)$ for all $i \in N \backslash\{\sigma(h+1), \sigma(h+2)\}$. Furthermore, $l_{\sigma(h+1)}^{\sigma}(v) \geq$ $l_{\sigma(h+1)}^{\sigma_{h+1}}(v)$ implying that

$$
\sum_{i \in S} l_{i}^{\sigma}(v) \geq \sum_{i \in S} l_{i}^{\sigma_{h+1}}(v) \geq v(S)
$$

where the first inequality is an equality if $\sigma(h+2) \in S$.

Case 3b. $\sigma(h+1) \notin S$. Then since $l_{\sigma(h)}^{\sigma}(v) \geq l_{\sigma(h)}^{\sigma_{h}}(v)$ and $l_{i}^{\sigma}(v)=l_{i}^{\sigma_{h}}(v)$ for every $i \in N \backslash\{\sigma(h), \sigma(h+1)\}$ we have

$$
\sum_{i \in S} l_{i}^{\sigma}(v) \geq \sum_{i \in S} l_{i}^{\sigma_{h}}(v) \geq v(S)
$$

Combining all three cases yields $l^{\sigma}(v) \in C(v)$.

The following theorem is a straightforward consequence of Lemma 3.1.

Theorem 3.1. Let $v \in T U^{N}, n \geq 3$. Then the following statements are equivalent:

1. $v$ is compromise stable,

2. $l^{\sigma}(v) \in C(v)$ for all odd $\sigma \in \Pi(N)$, 
3. $l^{\sigma}(v) \in C(v)$ for all even $\sigma \in \Pi(N)$.

In the remaining part of this section we present a necessary and sufficient condition for a set of orderings $A \subseteq \Pi(N)$ to be c-complete. Based on this condition, we are able to determine the minimum cardinality of c-complete sets. First, however, we provide some notation.

Let $P(N \backslash S, S)$ denote the set of orderings that begins with the players of $N \backslash S$ and ends with the players of $S$, i.e., $\sigma \in P(N \backslash S, S)$ if $\sigma(i) \in S$ for all $i \in$ $\{n-|S|+1, \ldots, n\}$. Furthermore, let $\overline{\mathcal{P}}=(P(N \backslash S, S))_{S, 1<|S|<n-1}$ be a collection of such sets. Observe that for any $k \in\{1, \ldots, n\}, \bigcup_{|S|=k} P(N \backslash S, S)=\Pi(N)$.

Example 3.1. Consider $N=\{1,2,3,4,5\}$. Then an element of $\overline{\mathcal{P}}$ is a set $P(N \backslash S, S)$ for some specific $S$. For example, for $S=\{1,2,4\}$,

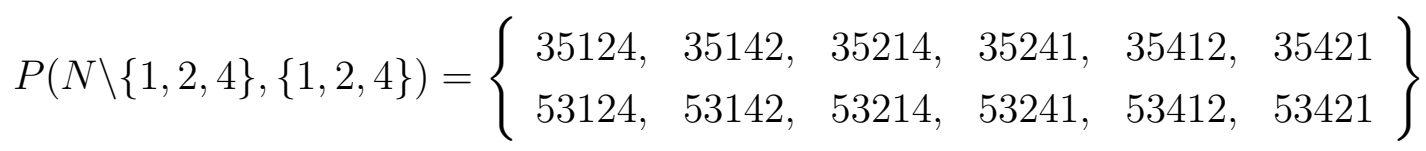

is an element of $\overline{\mathcal{P}}$ containing 12 orderings.

Theorem 3.2. A set $A \subseteq \Pi(N)$ is c-complete if and only if

$$
A \cap P(N \backslash S, S) \neq \emptyset \text { for all } P(N \backslash S, S) \in \overline{\mathcal{P}} \text {. }
$$

Proof. First we prove the 'if' part. Let $A \subset N$ be such that (3.1) holds, and let $(N, v) \in C A^{N}$. Assume that $l^{\sigma}(v) \in C(v)$ for all $\sigma \in A$. We will show that $A$ is c-complete by showing that $(N, v)$ is compromise stable. To do so, it is sufficient to show that the inequality in Theorem 2.1 (iv) is satisfied for all $S$. Observe that if $S=N, S=N \backslash\{i\}$ or $S=\{i\}$ with $i \in N$, then this inequality is satisfied.

Let $S \in 2^{N}$ with $1<|S|<n-1$. Take $\sigma \in A \cap P(N \backslash S, S)$. Considering the corresponding larginal, $l^{\sigma}(v)$, we distinguish between two cases.

Case 1. The pivot player of $l^{\sigma}(v)$ is in $N \backslash S$. This implies $\sum_{i \in S} l_{i}^{\sigma}(v)=\sum_{i \in S} m_{i}(v)$, and thus,

$$
v(S) \leq \sum_{i \in S} l_{i}^{\sigma}(v)=\sum_{i \in S} m_{i}(v),
$$

where the inequality holds since $l^{\sigma}(v) \in C(v)$. 
Case 2. The pivot player of $l^{\sigma}(v)$ is in $S$. This implies $l_{i}^{\sigma}(v)=M_{i}(v)$ for all $i \in N \backslash S$. Therefore,

$$
v(S) \leq \sum_{i \in S} l_{i}^{\sigma}(v)=v(N)-\sum_{i \in N \backslash S} l_{i}^{\sigma}(v)=v(N)-\sum_{i \in N \backslash S} M_{i}(v)
$$

where the inequality follows since $l_{i}^{\sigma}(v) \in C(v)$.

Combining (3.2) and (3.3) yields that

$$
v(S) \leq \max \left\{\sum_{i \in S} m_{i}(v), v(N)-\sum_{i \in N \backslash S} M_{i}(v)\right\}
$$

We conclude that $v$ is compromise stable, and therefore, $A$ is c-complete.

Second, we prove the 'only if' part. We show that $A$ is not c-complete if (3.1) is not satisfied, by providing a game such that all larginals corresponding to orderings in $A$ are core elements while the game is not compromise stable.

Assume that A does not fulfill (3.1). Then there exists a coalition $S^{*} \subset N, 1<$ $\left|S^{*}\right|<n-1$ such that $A \cap P\left(N \backslash S^{*}, S^{*}\right)=\emptyset$. Define the game $(N, v)$ by:

$$
v(T)=\left\{\begin{array}{cl}
1 & \text { if } T=S^{*} \\
0 & \text { if }|T| \leq\left|S^{*}\right|, T \neq S^{*} \\
|T|-\left|S^{*}\right| & \text { if }|T|>\left|S^{*}\right|
\end{array}\right.
$$

Note, that the utopia demand and minimum right will be $M_{i}(v)=1$ and $m_{i}(v)=0$ respectively for all $i \in N$. Then, for each $\sigma \in \Pi(N)$ the larginal $l^{\sigma}(v)$ becomes

$$
l_{\sigma(h)}^{\sigma}(v)= \begin{cases}1 & \text { for all } h \in\left\{1, \ldots, n-\left|S^{*}\right|\right\} \\ 0 & \text { for all } h \in\left\{n-\left|S^{*}\right|+1, \ldots, n\right\}\end{cases}
$$

First, we show that $l^{\sigma}(v) \in C(v)$ for all $\sigma \in A$.

Let $\sigma \in A$. We have to show that

$$
\sum_{i \in T} l_{i}^{\sigma}(v) \geq v(T)
$$

for all $T \in 2^{N} \backslash\{\emptyset, N\}$. Let $T \in 2^{N} \backslash\{\emptyset, N\}$. 
If $|T| \leq\left|S^{*}\right|, T \neq S^{*}$ then $v(T)=0$ and since $l_{i}^{\sigma}(v) \geq 0$ for all $i \in N$, (3.6) follows immediately.

If $T=S^{*}$, then $v(T)=1$ and since $\sigma \notin P\left(N \backslash S^{*}, S^{*}\right)$ at least one player in $T$ is at position $h$, with $h \in\left\{1, \ldots, n-\left|S^{*}\right|\right\}$, and again (3.6) holds.

If $|T|>\left|S^{*}\right|$, then $v(T)=|T|-\left|S^{*}\right|$, and by (3.5), (3.6) holds. Hence, $l^{\sigma}(v) \in C(v)$. Second, we show that $v$ is not compromise stable. Let $\tau \in P\left(N \backslash S^{*}, S^{*}\right)$. Then

$$
\sum_{i \in S^{*}} l_{i}^{\tau}(v)=0<v\left(S^{*}\right)=1
$$

hence $l^{\tau}(v) \notin C(v)$ and $v$ is not compromise stable. Thus $A$ is not c-complete.

According to Theorem 3.2, a set A of orderings can only be c-complete if for each $S \subset N$ with $1<|S|<n-1$ there exists an ordering $\sigma \in A$ such that $T_{|S|}^{\sigma}=S$. Note, that there may or may not exist orderings $\sigma \in A$ such that $T_{|S|}^{\sigma}=S$ for $S$ with $|S| \in\{1, n-1\}$. As an illustration of a c-complete set, consider the following example.

Example 3.2. Let $N=\{1,2,3,4\}$. An example of a c-complete set is $A=\{1234,1324,1423,2314,2413,3412\}$ since every coalition of size 2 is contained in the 2-tail of some ordering in $A$. Thus, $A$ is c-complete, according to (3.1). However, much larger sets of orderings may not be c-complete. Consider for example the set $B=\Pi(N) \backslash\{3412,3421,4312,4321\}$. This set contains 20 orderings, but it cannot be a c-complete set, since the coalition $\{1,2\}$ is not contained in the 2 -tail of any ordering in $B$.

The above example illustrates that even large sets of orderings with corresponding larginals in the core may not be c-complete. An upper bound on the cardinality of sets that are not c-complete is given in the proposition below.

Proposition 3.1. Let $A \subseteq \Pi(N)$ be a set of orderings with $|A|>n !-\left\lceil\frac{n}{2}\right\rceil !\left(n-\left\lceil\frac{n}{2}\right\rceil\right)$ !. Then $A$ is c-complete.

Proof. For any set of players $S \in \mathcal{S}_{k}$ we have $|P(N \backslash S, S)|=(n-k) ! k$ ! , i.e., there exist $(n-k) ! k$ ! different orderings $\sigma \in \Pi(N)$ such that $S=T_{k}^{\sigma}$. Since $|P(N \backslash S, S)| \geq$ $\left\lceil\frac{n}{2}\right\rceil !\left(n-\left\lceil\frac{n}{2}\right\rceil\right)$ ! for any $S \subset N$, it holds that $A \cap P(N \backslash S, S) \neq \emptyset$ for all $S \subseteq N$. Thus, $A$ is c-complete. 
Next, we focus on the minimum cardinality of a c-complete set of larginals defined as follows:

$$
L_{n}=\min \{|A|: A \subseteq \Pi(N) \text { is c-complete }\}
$$

Before we proceed, consider the collection of sets $\mathcal{P}=(P(N \backslash S, S))_{S,|S| \geq 1}$. Obviously, a set $A$ of orderings is also c-complete if $A \cap P(N \backslash S, S) \neq \emptyset$ for all $P(N \backslash S, S) \in$ $\mathcal{P}$.

Let a partial order on $\mathcal{P}$ be defined in the following way. $P\left(N \backslash S_{1}, S_{1}\right)<$ $P\left(N \backslash S_{2}, S_{2}\right)$ if $S_{1} \subset S_{2}$. Two elements $a, b$ of the partially ordered set $\mathcal{P}$ are said to be comparable if either $a \leq b$ or $b \geq a$. If neither $a \leq b$ nor $b \geq a$, then the two elements are said to be incomparable. Furthermore, let a set of pairwise comparable elements in $\mathcal{P}$ be denoted a chain, and let a set of pairwise incomparable elements be denoted an antichain.

A chain is then a sequence of sets $\left\{P\left(N \backslash S_{i_{1}}, S_{i_{1}}\right), P\left(N \backslash S_{i_{2}}, S_{i_{2}}\right), \ldots, P\left(N \backslash S_{i_{j}}, S_{i_{j}}\right)\right\}$ for $i_{1}, i_{2}, \ldots, i_{j} \in\{1, \ldots, n\}$ such that $S_{i_{1}} \subset S_{i_{2}} \subset \ldots \subset S_{i_{j}}$. A maximal chain is a chain that is not a proper subset of any other chain.

Furthermore, observe that each ordering $\sigma \in \Pi(N)$ corresponds to a maximal chain in $\mathcal{P}$. Let $\sigma=\{\sigma(1), \ldots, \sigma(n)\} \in \Pi(N)$. Then we can construct a maximal chain from the inclusion by choosing the sequence of sets such that

$$
S_{i_{1}} \subset S_{i_{2}} \subset \ldots \subset S_{i_{j}}=\{\sigma(1)\} \subset\{\sigma(1), \sigma(2)\} \subset \ldots \subset\{\sigma(1), \sigma(2), \ldots, \sigma(n)\} .
$$

Thus, we can represent any maximal chain by an ordering and vice versa.

Now we are ready to state the result.

Theorem 3.3. For $n \geq 3$, the minimum cardinality of a c-complete set is $L_{n}=\left(\begin{array}{c}n \\ \left\lceil\frac{n}{2}\right\rceil\end{array}\right)$. Proof. First, we note that there exists an antichain $B$ in $\mathcal{P}$ of cardinality $\left(\begin{array}{c}n \\ \left\lceil\frac{n}{2}\right\rceil\end{array}\right)$, namely the subset of $\mathcal{P}$ consisting of all $P(N \backslash S, S)$ with $|S|=\left\lceil\frac{n}{2}\right\rceil$. Since any c-complete set must intersect every element of this antichain, at least $\left(\begin{array}{c}n \\ \left\lceil\frac{n}{2}\right\rceil\end{array}\right)$ orderings are needed for $A$ to be c-complete. Next, we show that we can find a set of orderings $A$ of cardinality $\left(\begin{array}{l}n \\ \frac{n}{2}\end{array}\right)$ that intersects every element of the partially ordered set $\mathcal{P}$. Such a set must be c-complete.

According to Spernser's Theorem no antichain can have cardinality larger than 
$\left(\begin{array}{c}n \\ \left\lceil\frac{n}{2}\right\rceil\end{array}\right)$ (cf. Frankl (1995)), and $B$ is therefore a maximal antichain. Then, it follows from Dilworth's Theorem, that $\mathcal{P}$ can be decomposed into a set of chains $C$, with $|C|=\left(\begin{array}{l}n \\ \frac{n}{2}\end{array}\right)$ (cf. Trotter (1995)). Now, let $A$ be a set of orderings, such that for each chain $C_{i} \in C$ there exist an ordering in $A$ corresponding to $C_{i}$. Then, $A$ has cardinality $\left(\begin{array}{l}n \\ \frac{n}{2}\end{array}\right)$, and since it intersects every element of $\mathcal{P}, A$ is c-complete.

The following example illustrates the construction of a c-complete set of minimum cardinality.

Example 3.3. Let $N=\{1,2,3,4\}$. Any subset of $\mathcal{P}$ consisting of all elements corresponding to a given cardinality of $S$ is an example of an antichain. Thus, $B=$ $\{P(N \backslash\{1,2\},\{1,2\}), P(N \backslash\{1,3\},\{1,3\}), P(N \backslash\{1,4\},\{1,4\}), P(N \backslash\{2,3\},\{2,3\}), P(N \backslash$ $\{2,4\},\{2,4\}), P(N \backslash\{3,4\},\{3,4\})\}$ is an antichain in $\mathcal{P}$ of cardinality $\left(\begin{array}{l}4 \\ \frac{4}{2}\end{array}\right)=6$. Then there exists a partition $C=C_{1}, \ldots, C_{6}$ of $\mathcal{P}$ into 6 chains. A chain in $C$ is a sequence of sets of orderings like the following:

$P(N \backslash\{1\},\{1\}), P(N \backslash\{1,2\},\{1,2\}), P(N \backslash\{1,2,4\},\{1,2,4\}), P(N \backslash\{1,2,3,4\},\{1,2,3,4\})$,

and a partition of $\mathcal{P}$ could look like:

$$
\begin{aligned}
& C_{1}=P(N \backslash\{1\},\{1\}) \quad P(N \backslash\{1,2\},\{1,2\}) \quad P(N \backslash\{1,2,4\},\{1,2,4\}) \quad P(N \backslash\{1,2,3,4\},\{1,2,3,4\}) \\
& C_{2}=P(N \backslash\{1\},\{1\}) \quad P(N \backslash\{1,3\},\{1,3\}) \quad P(N \backslash\{1,3,4\},\{1,3,4\}) \quad P(N \backslash\{1,2,3,4\},\{1,2,3,4\}) \\
& C_{3}=P(N \backslash\{1\},\{1\}) \quad P(N \backslash\{1,4\},\{1,4\}) \quad P(N \backslash\{1,3,4\},\{1,3,4\}) \quad P(N \backslash\{1,2,3,4\},\{1,2,3,4\}) \\
& C_{4}=P(N \backslash\{2\},\{2\}) \quad P(N \backslash\{2,3\},\{2,3\}) \quad P(N \backslash\{1,2,3\},\{1,2,3\}) \quad P(N \backslash\{1,2,3,4\},\{1,2,3,4\}) \\
& C_{5}=P(N \backslash\{2\},\{2\}) \quad P(N \backslash\{2,4\},\{2,4\}) \quad P(N \backslash\{1,2,4\},\{1,2,4\}) \quad P(N \backslash\{1,2,3,4\},\{1,2,3,4\}) \\
& C_{6}=P(N \backslash\{3\},\{3\}) \quad P(N \backslash\{3,4\},\{3,4\}) \quad P(N \backslash\{1,3,4\},\{1,3,4\}) \quad P(N \backslash\{1,2,3,4\},\{1,2,3,4\})
\end{aligned}
$$

Based on the chains in $C$ we can now choose a set $A$ of orderings, such that each ordering in $A$ corresponds to a chain in $C$. We get $A=\{1243,1342,1432,2314,2413,3412\}$. We can easily check that $A$ is in fact c-complete by noting that every possible subset of $N$ of size 2 is contained in the tail of an ordering in $A$. 


\section{References}

Berge, C. (1973). Graphs and Hypergraphs. North-Holland, Amsterdam.

Curiel, I.J., M. Maschler, and S.H. Tijs (1988). Bankruptcy games. Zeitschrift fur Operations Research, 31, 143-159.

Driessen, T. (1988). Cooperative games, solutions and applications. Kluwer Academic Publishers, Dordrecht, The Netherlands.

Frankl, P. (1995). Extremal Set Systems, in R. L. Graham, M. Grotschel, and L. Lovasz (eds.), Handbook of Combinatorics, pp. 1293-1329. Elsevier

Gillies, D. (1953). Some theorems on n-person games. Ph. D. thesis, Princeton University Press, Princeton, New Jersey.

Ichiishi, T. (1981). Super-Modularity: Applications to Convex Games and the Greedy Algorithm for LP, Journal of Economic Theory, 25, 283-286.

König, D. (1931). Gráfok és mátrixok. Matematikai és Fizikai Lapok, 38, 116-119.

Muto, S., M. Nakayama, J. Potters, and S.H. Tijs (1988). On big boss games. Economic Studies Quarterly, 39, 303-321.

Potters, J. , R. Poos, S. Muto, and S.H. Tijs (1989). Clan games. Games and Economic Behaviour, 1, 275-293.

Quant, M., Borm, P., Reijnierse, H. and S. van Velzen (2005). The core cover in relation to the nucleolus and the weber set. Interna- 
tional Journal of Game Theory, 33, 491-503.

Rafels, C. and N. Ybern (1995). Even and odd marginal worth vectors, Owen's multilinear extension and convex games, International Journal of Game theory, 24, 113-126.

Shapley, L.S. (1971). Cores of convex games. International Journal of Game Theory, 1, 11-26.

Schmeidler D. (1969). The nucleolus of a characteristic function game. Siam Journal of Applied Mathematics, 17, 1163-1170.

Tijs and Lipperts (1982). The hypercube and the core cover of $n$ person cooperative games. Cahiers du Centre d'Études de Recherche Opérationelle, 24, 27-37.

Trotter, W.T. (1995). Partially Ordered Sets, in R.L.Graham, M. Grøtschel and L. Lovsz (eds.) Handbook of Combinatorics, p. 435-480. Elsevier.

Velzen,van B., H. Hamers, and H. Norde (2002). Convexity and marginal vectors, International Journal of Game theory, 31, 323-330.

Velzen,van B., H. Hamers, and H. Norde (2004). Characterizing convexity of games using marginal vectors, Discrete Applied Mathematics, 143, 298-306.

Weber, R.J. (1988). Probabilistic values of games. In A.E. Roth (Ed.), The Shapley value, 101-119. Cambridge University Press, Cambridge. 